\title{
Sixty-Four-Slice Multidetector Computerized Tomography in the Evaluation of Transmesenteric Internal Hernias following Roux-en-Y Bariatric Surgery
}

\author{
Osama Al-Saeed $^{a}$ Dalia Fahmy $^{b}$ Osama Kombar $^{\text {b, c }}$ Ali Hasan $^{d}$ \\ aDepartment of Radiology, Faculty of Medicine, Health Sciences Centre, Kuwait University, Kuwait City, Kuwait; \\ ${ }^{\mathrm{b}}$ Department of Radiology, Mansoura University, Mansoura, Egypt; Departments of ${ }^{\mathrm{C}}$ Radiology and ${ }^{\mathrm{d}}$ Surgery, \\ Amiri Hospital, Kuwait City, Kuwait
}

\section{Key Words}

Internal hernia · Bariatric surgery · Computerized

tomography $\cdot$ Roux-en-Y $\cdot$ Gastric bypass

\begin{abstract}
Objective: To evaluate the accuracy of 64-slice multidetector computerized tomography (MDCT) in the detection of transmesenteric internal hernias in patients following Rouxen-Y gastric bypass (RYGB) for bariatric surgery patients. Subjects and Methods: This retrospective study was performed on post-bariatric RYGB patients presenting with signs and symptoms suggestive of internal hernias at our institution from the period of April 2010 until March 2012. The patients who had symptoms suggestive of internal hernia had undergone 64-slice MDCT. All the patients who on CT examination were found to have features suggestive of internal hernia were subjected to laparoscopic exploration. Results: Of the 102 patients who had undergone laparoscopic RYGB, 42 (41.2\%) were suspected of having internal hernia. Of these, $23(55 \%)$ had CT findings of hernia while the remaining 19 (45\%) were considered normal. Of the 23,21 (91\%) patients were confirmed for internal hernia at laparoscopy. The 19 (45\%) patients that did not reveal any signs for internal hernia on CT and the 2 patients that were consid-
\end{abstract}

ered normal on laparoscopy were treated conservatively. The sensitivity, specificity and positive and negative predictive values for MDCT in the diagnosis of internal hernias were $100,90.5,91$ and $91.3 \%$ respectively. Conclusion: The 64 -slice MDCT was accurate in the diagnosis of transmesenteric internal hernias in post-RYGB for bariatric surgery patients. The presence of clustered loops with mesenteric swirl is a reliable indicator of transmesenteric internal hernia.

Copyright $\odot 2013$ S. Karger AG, Basel

\section{Introduction}

Obesity is a recognized major health problem and its prevalence, especially in the advanced countries and affluent societies, is increasing $[1,2]$. Kuwait is now considered as having one of the highest percentages of obesity just second to the USA [3]. Nonsurgical treatments for morbid obesity have limited long-term success and bariatric surgery has increased since it has been shown to be the only method that can provide long-term weight loss [4]. Various restrictive procedures have gained popularity but are associated with a higher long-term failure and complications [5]. The highest long-term success rates have been reported with Roux-en-Y gastric bypass (RYGB) [2], but

\begin{tabular}{ll}
\hline KARGER & $\begin{array}{l}\text { ○ 2013 S. Karger AG, Basel } \\
1011-7571 / 13 / 0226-0540 \$ 38.00 / 0 \quad \text { Karger }\end{array}$ \\
E-Mail karger@karger.com & $\begin{array}{l}\text { This is an Open Access article licensed under the terms of the } \\
\text { Creative Commons Attribution-NonCommercial 3.0 Un- } \\
\text { ported license (CC BY-NC) (www.karger.com/OA-license), } \\
\text { applicable to the online version of the article only. Distribu- } \\
\text { tion permitted for non-commercial purposes only. }\end{array}$
\end{tabular}

Dr. Osama Al-Saeed, ABR, Associate Professor and Consultant Radiologist Radiology Department, Faculty of Medicine Health Sciences Centre, Kuwait University PO Box 24923, Safat 13110 Kuwait (Kuwait) E-Mail osamas@ hsc.edu.kw 
it involves a major surgical procedure with high early morbidity. The RYGB surgery, despite being a major demanding surgical procedure, is the treatment of choice for satisfactory weight loss in revisional surgery [6]. Internal hernias are a recognized complication of the RYGB procedure and delay in diagnosis of any type of internal hernia can have catastrophic outcome if not recognized and treated promptly [7]. It has been noted that internal hernias are difficult to diagnose with both clinical and imaging studies [6]. However, CT scanning of the abdomen is the first-line imaging technique in these patients.

This study aimed to evaluate the accuracy of 64-slice multidetector computerized tomography (MDCT) in detecting internal hernias in post-laparoscopic RYGB bariatric surgery.

\section{Subjects and Methods}

This is a retrospective study on consecutive patients referred to the Department of Radiology, Amiri Hospital, Kuwait from April 2010 to March 2012 for CT examination of the abdomen and pelvis to identify internal hernia in patients who had undergone laparoscopic RYGB-type bariatric surgery. The inclusion criteria were patients with symptoms suggestive of internal hernia such as colicky abdominal pain, nausea, vomiting and other signs of bowel obstruction that did not resolve with conservative therapy. The exclusion criteria were patients who were found to have other reasons for their symptoms such as gallbladder or renal stones, pelvic diseases (e.g. ovarian) or medical conditions such as gastroenteritis. Appropriate permission from the institution was obtained to review patient records for the study.

\section{MDCT Protocol}

CT examinations had been performed using a 64-slice MDCT scanner (high-speed QX/i; GE Medical Systems, Milwaukee, Wisc., USA) with a gantry rotation speed of $0.8 \mathrm{~s}$ per rotation. The scanning parameters were coverage area from the diaphragm to the symphysis pubis scanned in cranio-caudal direction, slice thickness of $5 \mathrm{~mm}$, pitch of 1.5, table speed of $15 \mathrm{~mm}$ per rotation, reconstruction interval of $2 \mathrm{~mm}$, tube voltage of $120 \mathrm{kV}$ and tube current of $200 \mathrm{~mA}$. The MDCT scans of the abdomen and pelvis were performed before and after intravenous injection of non-ionic contrast medium during one breath-hold, from the dome of the liver to the lower margin of the symphysis pubis. The technique was accomplished using 120-150 ml of non-ionic contrast media with a flow rate of $3 \mathrm{ml} / \mathrm{s}$, which was administered via an 18-gauge intravenous cannula in the antecubital vein by means of an automatic injector (Medrad Stellant CT Injector, Warrendale, Pa., USA). The scan delay was determined using automated bolus-triggering software (Smart Prep; GE Healthcare, Milwaukee, Wisc., USA) program. The region of interest cursor was positioned in the descending aorta $2 \mathrm{~cm}$ above the diaphragm. Two scans were performed, the first one after $45 \mathrm{~s}$ delay and the second one after $70 \mathrm{~s}$ delay. A delayed scan after 7-10 min was also done if needed. Scans were reviewed as digital images on a picture archiving and com-
Table 1. Presenting signs and symptoms of patients included in this study, who were positive for transmesenteric internal hernia by CT and laparoscopy

\begin{tabular}{ll}
\hline Sign or symptom & $\begin{array}{l}\text { Number of } \\
\text { patients }(\mathrm{n}=21)\end{array}$ \\
\hline Abdominal pain & 21 \\
Nausea and vomiting & 15 \\
Abdominal tenderness & 7 \\
Guarding & 5 \\
Recurrent intermittent abdominal pain & 4 \\
Colicky pain referred to the back & 9 \\
Abdominal distension & 13 \\
Pyrexia & 2 \\
Leucocytosis & 1 \\
\hline
\end{tabular}

munication system workstation monitor (IMPAX DS3000; AGFA, Mortsel, Belgium). Coronal and sagittal multiplanar images were reconstructed.

All the patients who were diagnosed with internal hernia based on CT features were subjected to laparoscopic surgery which served both to confirm the diagnosis and for therapeutic procedure.

Two radiologists (O.A. and O.K.) with more than 15 years of experience in abdominal imaging, who were blinded to the surgical outcome and original pathology report, independently evaluated the images and differences, if any, were settled by consensus. Statistical analysis was done to determine the sensitivity, specificity and positive and negative predictive values for MDCT in the diagnosis of internal hernias.

\section{Results}

A total of 102 patients had post-RYGB abdominal symptoms. Because 42 patients ( 10 males and 32 females) satisfied the inclusion criteria, they had CT examination. Of these, 23 (55\%; 6 males and 17 females) had CT features suggestive of internal hernia while the remaining 19 (45\%) were considered normal. Of these 23 patients, 21 (91\%; 4 males and 17 females) were proved positive by laparoscopy and the other 2 (9\%) patients were normal. The 19 patients who were considered normal on CT examination and the 2 patients who were false positive on laparoscopy improved with conservative therapy. The sensitivity, specificity and positive and negative predictive values for MDCT in the diagnosis of internal hernias were 100, 90.5, 91 and $91.3 \%$, respectively.

The various signs and symptoms presented by the patients are shown in table 1 . The most common symptom noticed was abdominal pain followed by nausea and vomiting. The time interval from the initial abdominal 


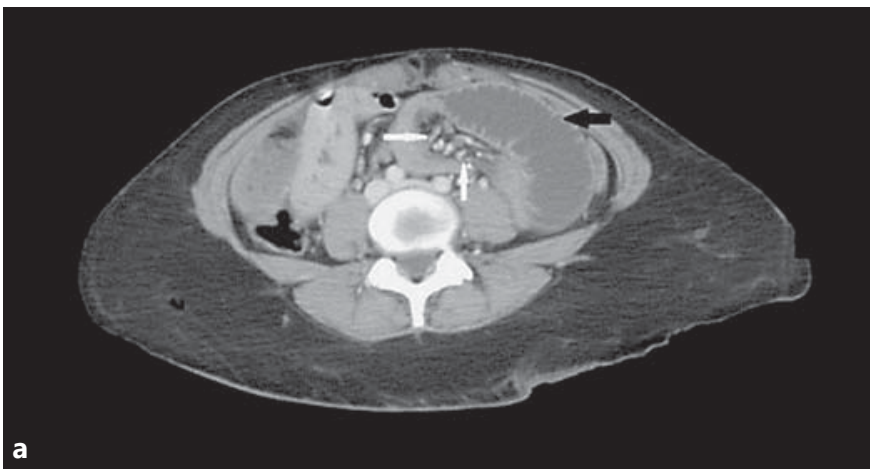

Fig. 1. A 32-year-old female with post-gastric bypass in 2008 presented with repeated vomiting and severe abdominal pain. a Dilated fluid-filled small-bowel loop is seen at the left side of the upper and middle abdomen (black arrow) and is associated with

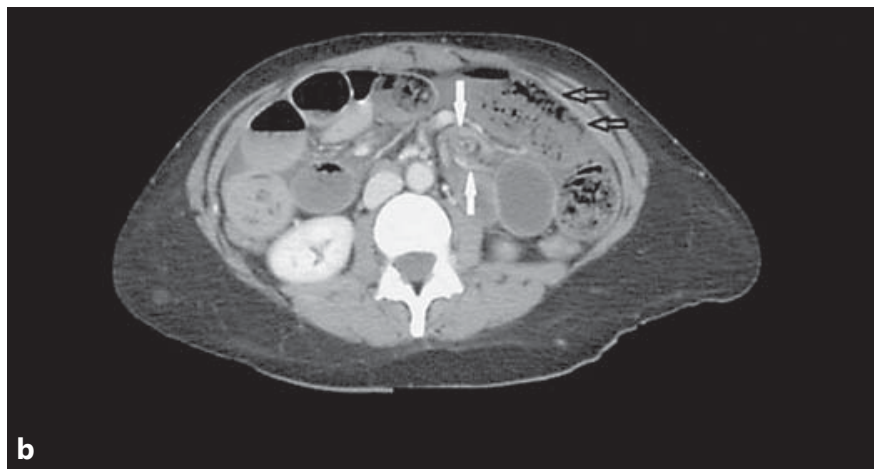

engorged straightened mesenteric vessels (white arrows). b Mesenteric swirl (white arrows) and anteriorly located small-bowel loops with no covering of omental fat (double open black arrows).
Table 2. Computerized tomography signs suggestive of internal hernia confirmed by laparoscopy in 21 patients

\begin{tabular}{ll}
\hline Sign & $\begin{array}{l}\text { Number of } \\
\text { patients }\end{array}$ \\
\hline
\end{tabular}

Clustered loops

21

Crowding of mesenteric vessels

Engorged, straightened vessels

Swirled appearance of mesenteric fat or vessels

Abnormal anterior location with loss of omental covering

Mushroom shape of mesenteric root

Tubular distal mesenteric fat

Displacement of colon centrally by small intestinal loops

Mesenteric ascites

Dilated loops suggesting obstruction tion -4 of these were partial obstruction and 1 showed ischemic changes of the obstructed bowel loops.

Of the 102 patients, 60 (59\%) who did not fit into the inclusion criteria for CT examination were followed up and treated according to their provisional diagnosis. None presented to us again with internal hernia symptoms.

\section{Discussion}

In this study, 64-slice MDCT proved to be very accurate in confirming or ruling out the diagnosis of internal hernia in patients who underwent RYGP-type bariatric surgery. This imaging procedure appears very valuable in guiding the surgeon to decide between urgent intervention and conservative treatment in patients with suspected internal hernia. Internal hernia following gastric bypass occurs when a bowel loop extends into an abnormal location through an opening in the mesentery. The overall incidence of internal hernias is reported as $0.2-0.9 \%$ [7] but in recent years higher incidence has been reported, about $3 \%$ compared with $<0.5 \%$ for open surgery (at a mean follow-up of 2 years), and this change is attributed to the increase in bariatric surgery which has grown steadily in response to the rising prevalence of obesity [8-11]. It has been suggested that patients are more prone to internal hernia after laparoscopic RYGB than after an open operation because there are fewer adhesions to tether small-bowel loops that prevent the bowel from herniation [12]. Higher incidence is reported in those subjects who have greater degrees of weight loss because of loss of the protective, space-occupying effect of mesenteric fat 


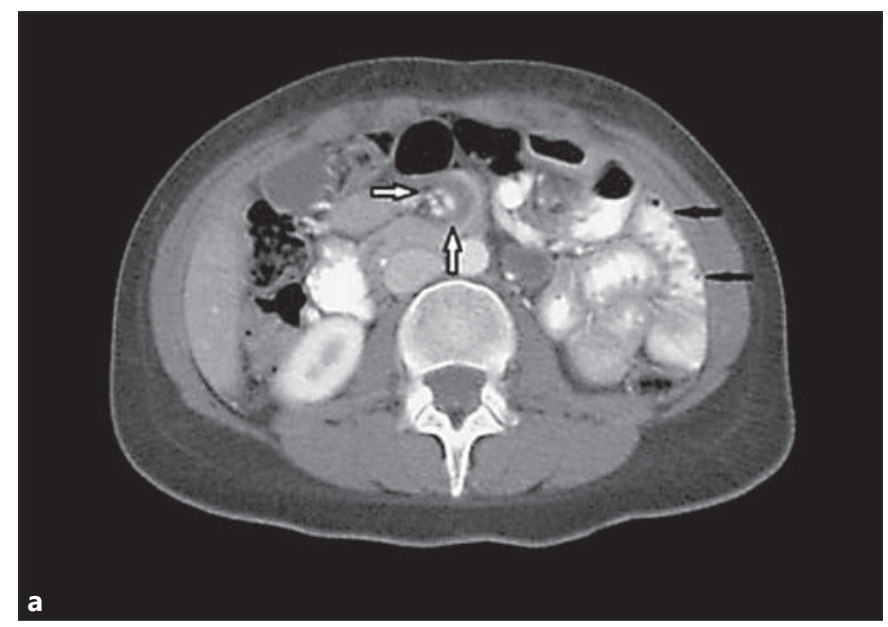

Fig. 2. A 33-year-old woman who had history of gastric bypass 7 years previously presented with abdominal pain and nausea. a Aggregation of small-bowel loops (jejunal) is seen along the left side of the abdomen showing mural enhancement (black arrows). This is associated with engorgement and swirling of the corre-

$[9,10]$. Though all the patients included in our study had laparoscopic RYGB, we were unable to determine the incidence of post-laparoscopic RYGB internal hernias in our population as no published data are available on bariatric surgery performed in Kuwait. This is the first published data regarding complications of bariatric surgery in our population. There is no age or sex predilection for internal hernias [5], but in this study a higher incidence was observed in females ( 17 of the 21 cases), probably because bariatric surgery is more popular among females.

Internal hernias are difficult to diagnose, both clinically as well as by imaging studies [6]. The presenting symptoms, as in our patients, are usually post-prandial abdominal pain, nausea and vomiting [13]. MDCT has evolved as the first-line imaging technique in these patients because of its speed and multiplanar reformatting capabilities [5]. The use of oral and intravenous contrast material is advocated for better delineation and localization of intestinal loops as well as mesenteric vessels and peritoneal cavity. Some researchers reported that oral administration of contrast material and water are not necessary in view of the patient's severe condition because intraluminal fluid collected within an obstructed bowel segment already serves as a natural contrast agent, demonstrating the bowel wall clearly [14], while others advocated the use of CT enteroclysis, which provides a flexible method of viewing small-bowel obstruction [15]. In our institution oral contrast material is routinely administered unless contraindi-

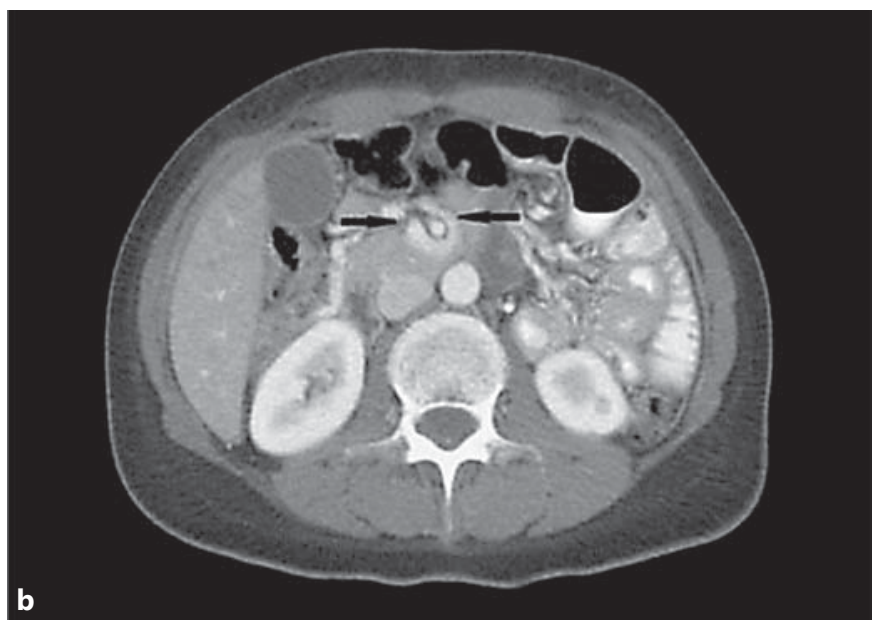

sponding mesenteric vessels (white arrows). b Mushroom sign, narrowed mesenteric root with passage of intestinal loops between superior mesenteric artery and its branches is clearly demonstrated (black arrows).

cated $[9,10]$. The most common features noticed on the CT examination of the abdomen in this study confirmed previous reports $[7-10,16,17]$ of clustered bowel loops, crowding and engorged and straightened mesenteric vessels, followed by swirled appearance of the mesenteric fat or vessels. The sensitivity (100\%), specificity $(90.5 \%)$, positive predictive value (91\%) and negative predictive value (91.3\%) in this study using mesenteric swirl and clustered bowel loop signs are together higher than the most widely reported study of Lockhart et al. [8] in which sensitivity was $83 \%$ and specificity $94 \%$ using swirl sign alone, indicating the impact of using more than one sign in making the diagnosis. Use of $3 \mathrm{D}$ and $2 \mathrm{D}$ reconstructed images that allowed better detection of swirl sign have been reported [18] as the best-performing single sign in the diagnosis of internal hernia. Peripheral location of the small loops in the abdominal cavity and the absence of omental fat between the bowel loops and the anterior abdominal wall are two other imaging features of internal hernia with reported sensitivity of 85 and $92 \%$, respectively [9]. These findings were noticed in $71 \%$ of the cases in our study, indicating that variable presentations are possible and may not be seen in all patients. The mushroom shape of the mesenteric root which refers to the shape of the hernia as it passes through a narrow opening between vessels at the base of the mesentery (fig. 2) was noticed in 33\% of cases of transmesenteric hernia, while tubular distal mesenteric fat was detected in $28 \%$ of the cases in our study. Distal tubu- 
lar mesenteric fat with closely surrounding bowel loops is similar to mesenteric swirl but includes small-bowel loops closely surrounding round or tubular mesenteric fat [8].

Blachar et al. [10], in a study on 16 cases of transmesenteric hernias reported that these hernias are usually not capsulated and are therefore difficult to detect. However, in a study by Blachar and Federle [18], it was reported that clustered loops of small bowel located lateral to the colon (a reversal of the normal pattern) with central, inferior or posterior displacement of the transverse colon act as a clue to mesenteric hernias. This feature was found in $26 \%$ of the cases in our study and none of the cases were capsulated. As observed by Lockhart et al. [8], the imaging features of ischaemic bowel changes concur with clinical signs of inflammation (elevated white blood cell count and guarding and diffuse abdominal tenderness) with increased morbidity and mortality. None of the cases in our study showed imaging features of bowel ischaemia, which may be attributed to quicker management secondary to more awareness of such complications in clinical practice as the rate of RYGP increased.
The major limitation of our study was the lack of data regarding the number of bariatric surgeries performed in our institutional catchment area, which led to our inability to determine the incidence of this complication in our population. This limitation could have affected the degree of sensitivity and specificity of the provisional clinical diagnosis, which could have a great impact on justifying the cost effectiveness and radiation risk involved with MDCT. It is important to carry out future studies on bariatric complications on a larger scale, including not only RYGB but also more recent procedures such as gastric sleeve surgeries.

\section{Conclusion}

The 64-slice MDCT was accurate in the diagnosis of transmesenteric internal hernias in post-RYGB for bariatric surgery patients. The presence of clustered loops with mesenteric swirl was a reliable indicator of transmesenteric internal hernia.

\section{References}

1 Fisher BL, Schauer P: Medical and surgical options in the treatment of severe obesity. Am J Surg 2002;184:9-16.

$>2$ Talieh J, Kirgan D, Fisher BL: Gastric bypass for morbid obesity: a standard surgical technique by consensus. Obes Surg 1997;7:198-202.

-3 Walpole SC, Prieto-Merino D, Edwards P, et al: The weight of nations: an estimation of adult human biomass. BMC Public Health 2012;12:439.

$\checkmark 4$ Kim TH, Daud A, Ude AO, et al: Early US outcomes of laparoscopic gastric bypass versus laparoscopic adjustable silicone gastric banding for morbid obesity. Surg Endosc 2006;20:202-209.

5 Martin LC, Merkle EM, Thompson WM: Review of internal hernias: radiographic and clinical findings. AJR Am J Roentgenol 2006; 186:703-717.

6 Ghiassi S, Nguyen SQ, Divino CM, et al: Internal hernias: clinical findings, management and outcomes in 49 nonbariatric cases. J Gastrointest Surg 2007;11:291-295.
7 Higa KD, Ho T, Boone KB: Internal hernias after laparoscopic Roux-en-Y gastric bypass: incidence, treatment and prevention. Obes Surg 2003;13:350-354.

8 Lockhart ME, Tessler FN, Canon CL, et al: Internal hernia after gastric bypass: sensitivity and specificity of seven CT signs with surgical correlation and controls. AJR Am J Roentgenol 2007; 188:745-750.

-9 Blachar A, Federle MP, Dodson SF: Internal hernia: clinical and imaging findings in $17 \mathrm{pa}$ tients with emphasis on CT criteria. Radiology 2001;218:68-74.

10 Blachar A, Federle MP, Brancatelli G, et al: Radiologist performance in the diagnosis of internal hernia by using specific CT findings with emphasis on transmesenteric hernia. Radiology 2001;221:422-428.

$>11$ Garza E Jr, Kuhn J, Arnold D, et al: Internal hernias after laparoscopic Roux-en-Y gastric bypass. Am J Surg 2004;188:796-800.

12 Champion JK, Williams M: Small bowel obstruction and internal hernias after laparoscopic Roux-en-Y gastric bypass. Obes Surg 2003;13:596-600.
13 Meyers MA: Dynamic Radiology of the Abdomen: Normal and Pathologic Anatomy, ed 4. New York, Springer, 1994.

14 Horton KM, Fishman EK: The current status of multidetector-row CT and three-dimensional imaging of the small bowel. Radiol Clin North Am 2003;41:199-212.

15 Maglinte DD, Bender GN, Heitkamp DE, et al: Multidetector-row helical CT enteroclysis. Radiol Clin North Am 2003;41:249262.

16 Takeyama N, Gokan T, Ohgiya Y, et al: CT of internal hernias. Radiographics 2005;25:9971015.

17 Mathieu D, Luciani A: Internal abdominal herniations. AJR Am J Roentgenol 2004;83: 397-404.

18 Blachar A, Federle MP: Gastrointestinal complications of laparoscopic Roux-en-Y gastric bypass surgery in patients who are morbidly obese: findings on radiography and CT. AJR Am J Roentgenol 2002;179:14371442 . 